\title{
Clumping and Viability of Bone Marrow Derived Mesenchymal Stromal Cells under Different Preparation Procedures: A Flow Cytometry-Based In Vitro Study
}

\author{
Li-li Cui, ${ }^{1}$ Tuure Kinnunen, ${ }^{2}$ Johannes Boltze, $, 3,4$ \\ Johanna Nystedt, ${ }^{6}$ and Jukka Jolkkonen ${ }^{1,7}$ \\ ${ }^{1}$ Institute of Clinical Medicine-Neurology, University of Eastern Finland, 70210 Kuopio, Finland \\ ${ }^{2}$ Department of Clinical Microbiology, Institute of Clinical Medicine, University of Eastern Finland, 70210 Kuopio, Finland \\ ${ }^{3}$ Fraunhofer Institute for Cell Therapy and Immunology, 04103 Leipzig, Germany \\ ${ }^{4}$ Fraunhofer Research Institution for Marine Biotechnology, 23562 Lübeck, Germany \\ ${ }^{5}$ Institute for Medical and Marine Biotechnology, University of Lübeck, 23562 Lübeck, Germany \\ ${ }^{6}$ Finnish Red Cross Blood Service, Advanced Cell Therapy Centre, 00310 Helsinki, Finland \\ ${ }^{7}$ Neurocenter, Neurology, University Hospital of Kuopio, 70210 Kuopio, Finland
}

Correspondence should be addressed to Jukka Jolkkonen; jukka.jolkkonen@uef.fi

Received 27 November 2015; Revised 15 January 2016; Accepted 4 February 2016

Academic Editor: Shinn-Zong Lin

Copyright (C) 2016 Li-li Cui et al. This is an open access article distributed under the Creative Commons Attribution License, which permits unrestricted use, distribution, and reproduction in any medium, provided the original work is properly cited.

Complications of microocclusions have been reported after intra-arterial delivery of mesenchymal stromal cells. Hence, quantification and efficient limitation of cell clumps in suspension before transplantation is important to reduce the risk. We used a flow cytometry-based pulse-width assay to assess the effects of different cell suspension concentrations $\left(0.2-2.0 \times 10^{6} / \mathrm{mL}\right)$, storage solutions (complete growth medium, Dulbecco's phosphate-buffered saline, and normal saline), storage time in suspension (0$9 \mathrm{~h}$ ), and freeze-thawing procedure on the clumping of rat bone marrow derived mesenchymal stromal cells (BMMSCs) and also evaluated cell viability at the same time. Surprisingly, increasing the cell concentration did not result in more cell clumps in vitro. Freshly harvested (fresh) cells in normal saline had significantly fewer cell clumps and also displayed high viability ( $>90 \%)$. A timedependent reduction in viability was observed for cells in all three storage solutions, without any significant change in the clumping tendency except for cells in medium. Fresh cells were more viable than their frozen-thawed counterparts, and fresh cells in normal saline had fewer cell clumps. In conclusion, cell clumping and viability could be affected by different cell preparation procedures, and quantification of cell clumping can be conducted using the flow cytometry-based pulse-width assay before intra-arterial cell delivery.

\section{Introduction}

Mesenchymal stromal cells (MSCs) can be isolated from various tissues such as bone marrow, umbilical cord blood, and adipose tissue. MSCs are promising candidates for cell therapy because of their multipotency, immunomodulatory effects, easy accessibility, lack of immunogenicity as well as their ethical advantages. Promising positive effects of MSCs administration have been obtained in experimental studies on stroke treatment [1-3] and some early phase clinical trials are currently in progress [4].
Intravascular MSC delivery has been most commonly used in both preclinical and clinical studies with the least invasiveness. However, after intravenous infusion, most cells have been found to be trapped in the internal organs [5], leading to a potential risk of pulmonary embolism [6]. Intra-arterial infusion can increase the cell homing to the ischemic hemisphere since this circumvents the pulmonary circulation $[7,8]$, but this route carries also a higher risk of complications such as microocclusions [9-12]. For example, in our previous study using allogeneic bone marrow derived mesenchymal stromal cells (BMMSCs), a dose-dependent 
cerebral embolism was evoked after intra-arterial cell delivery into rats [12]. The relatively large size of MSCs is one important reason for the vascular embolism after cell therapy $[11,13]$. Another possible reason is that cell clumps exist in suspension already prior to transplantation. To reduce the potential risk of embolism while maintaining efficacy, it is important to quantify cell clumping and limit the number of large clumps, but so far few studies have addressed this issue.

The flow cytometry-based pulse-width assay has been introduced as a rapid method with a high level of accuracy and sensitivity for quantifying cell clumps [14]. In addition, cell viability, an important in vitro predictor of the efficacy of cell therapy [15], can also be easily evaluated by flow cytometry. During the cell preparation procedure, many variables from ex vivo expansion until delivery might affect the tendency towards cell clumping as well as cell viability. It is very important that, before transplantation, one can be sure that there are limited cell clumps in the cell suspension which has maintained good cell viability. Therefore, we applied the flow cytometry-based assay to assess the effects of different cell suspension concentrations $\left(0.2-2.0 \times 10^{6} / \mathrm{mL}\right)$, different storage solutions (complete growth medium, Dulbecco's phosphate-buffered saline and normal saline), storage time in suspension (0-9h), and freeze-thawing procedure on cell clumping as well as cell viability.

\section{Materials and Methods}

2.1. Cell Culture and Characterization of Bone Marrow Derived Mesenchymal Stromal Cells. Oricell ${ }^{\mathrm{TM}}$ male Wistar rat BMMSCs (Cyagen Bioscience Inc., Cat. No. RAWMX-01001) were used in order to be consistent with our previous work [12]. According to the manufacturer's instructions, the cells were cultured in OriCell MSC growth medium supplemented with $10 \%$ fetal bovine serum (FBS), 1\% glutamine, and $1 \%$ penicillin-streptomycin (all reagents are from Cyagen Biosciences Inc., Cat. No. GUXMX-90011). The medium was changed twice every week. The cells were passaged after reaching $80-90 \%$ confluency and subcultured at a cell density of 6000 cells $/ \mathrm{cm}^{2}$. Rat BMMSCs at passage 5 were cryopreserved in the protein-free Oricell ${ }^{\mathrm{TM}}$ NCR cryopreservation medium (Cyagen Biosciences Inc., Cat. No. NCPF-10001). The cells were characterized as previously described [16].

2.2. Preparation of Cell Samples for Analysis. Before measurement, cultured rat BMMSCs at passage 5 were harvested using $0.05 \%$ trypsin-EDTA (Life Technologies, Cat. No. 25300-054); cryopreserved rat cells were thawed in a water bath at $37^{\circ} \mathrm{C}$ before being decanted into the complete medium (OriCell MSC growth medium supplemented with $10 \%$ FBS, $1 \%$ glutamine, and $1 \%$ penicillin-streptomycin). After centrifugation at $1000 \mathrm{~g}$ for $5 \mathrm{~min}$, freshly harvested (fresh) or frozen-thawed (thawed) cells were resuspended in Dulbecco's phosphate-buffered saline (DPBS) without calcium or magnesium, normal saline (NS; $0.9 \% \mathrm{NaCl}$ ), or complete medium. To analyze the effect of cell concentration on cell clumping and viability in the MSC suspension before transplantation, fresh cells were resuspended in DPBS at a

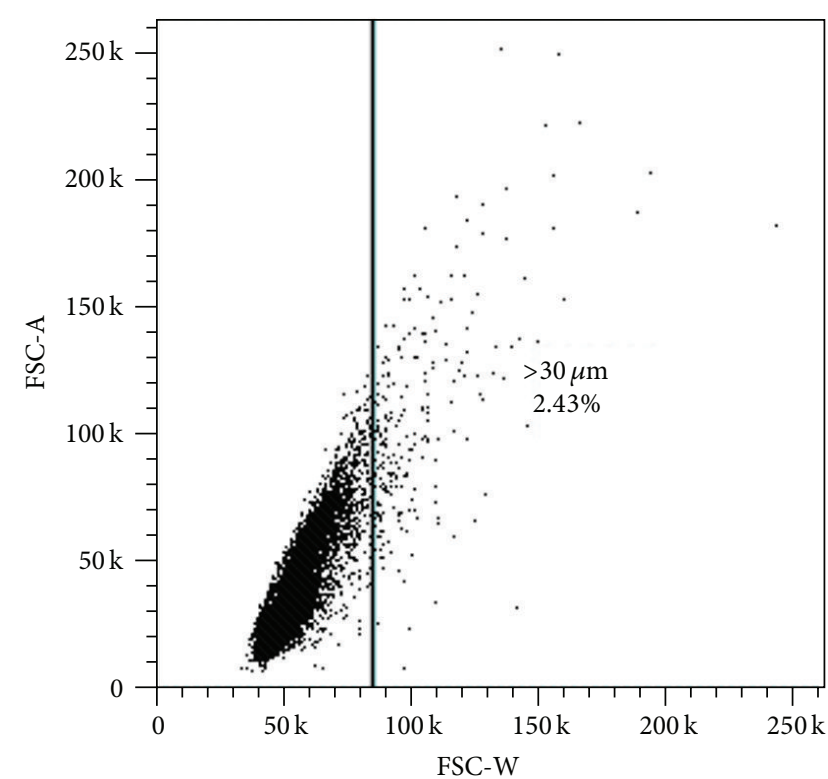

FIgURE 1: Gating of cell clumps or large cells $>30 \mu \mathrm{m}$. After the exclusion of debris, FSC-W was plotted against FSC-A and calibrated using standardized polystyrene microspheres.

concentration of $0.2 \times 10^{6} / \mathrm{mL}, 0.5 \times 10^{6} / \mathrm{mL}, 1.0 \times 10^{6} / \mathrm{mL}$, and $2.0 \times 10^{6} / \mathrm{mL}$, respectively. The effect of storage time was assessed by maintaining the cells at $37^{\circ} \mathrm{C}$ for $3 \mathrm{~h}, 6 \mathrm{~h}$, and $9 \mathrm{~h}$ at a concentration of $0.2 \times 10^{6} / \mathrm{mL}$ before measurement.

2.3. Detection of Cell Clumping Using the Flow CytometryBased Pulse-Width Assay. The pulse width is based on the laser beam height, particle velocity, and particle diameter. Because of the constant sheath pressure which ensures a stable particle velocity, and the constant beam height within the instrument setup, the pulse width can be directly correlated with the diameter of the particle. After calibration of the FSC-W axis by standardized polystyrene microspheres (Polysciences Inc., Cat. No. 64165-15), clumps or large cells $>30 \mu \mathrm{m}$ were gated as suggested by Hickerson et al. [14]. FSC$\mathrm{W}$ was plotted against FSC-A by adjusting the photomultiplier tube voltage and area scaling factor (Figure 1).

2.4. Detection of Cell Viability, Apoptosis, and Death by Flow Cytometry. Cell apoptosis and death were assessed with a CellEvent $^{\mathrm{Tm}}$ Caspase-3/7 Green Flow Cytometry Assay Kit (ThermoFisher Scientific, Cat. No. C10427) according to the manufacturer's instruction. Briefly, CellEvent ${ }^{\mathrm{Tm}}$ Caspase-3/7 Green Detection Reagent was added to the cell suspension and then incubated in the dark at $37^{\circ} \mathrm{C}$ for 30 minutes. After the final 5 minutes of staining, SYTOX ${ }^{\circledR}$ AADvanced $^{\mathrm{TM}}$ dead cell stain solution in dimethylsulfoxide was added to the suspension. The samples were run on a FACS Canto II flow cytometer (BD Biosciences, USA) using FACSDiva software. The data was analyzed using Flowjo 7.6.2 (Figure 2). Trypan blue staining was also used to determine cell numbers and cell viability in a Countess ${ }^{\mathrm{TM}}$ automated cell counter (Invitrogen, Cat. No. C10281). 


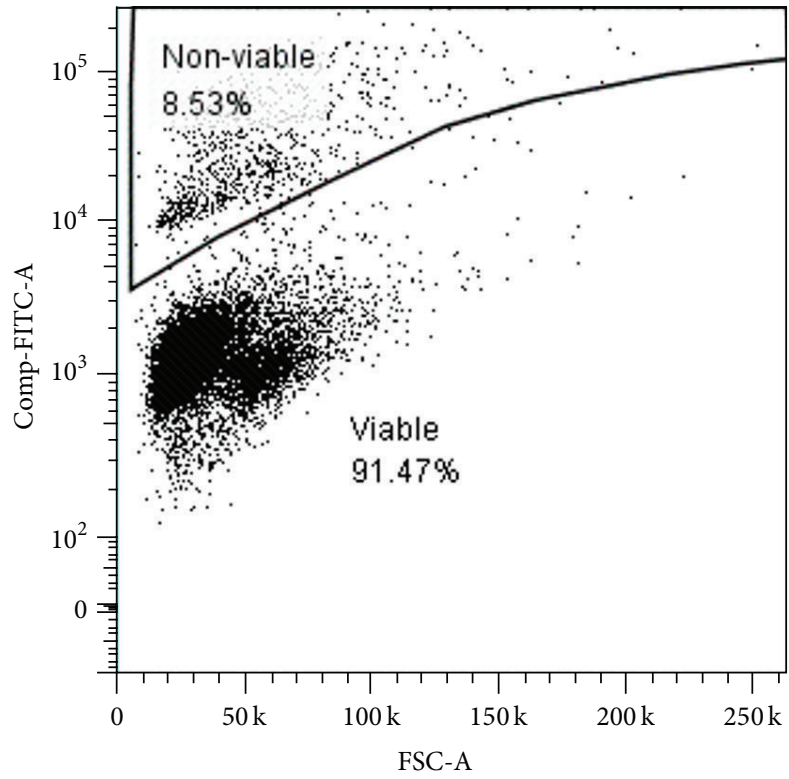

(a)

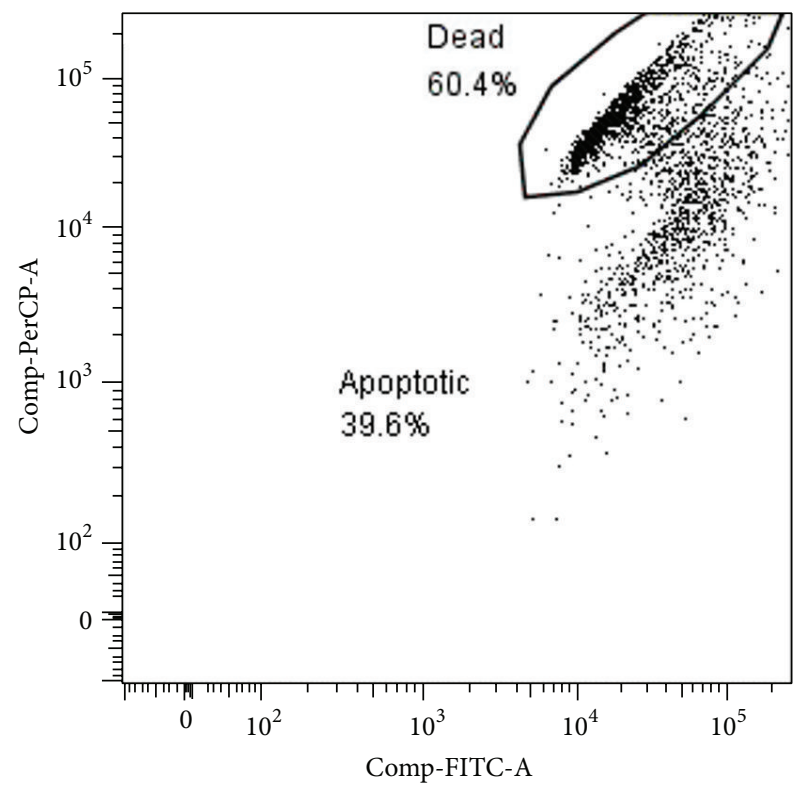

(b)

FIGURE 2: Gating of viable, dead, and apoptotic MSCs. (a) After excluding debris, the CellEvent ${ }^{\text {tm }}$ caspase-3/7 FITC positive cells were gated as nonviable, and the rest as viable cells. (b) Nonviable cells were then gated as dead (SYTOX ${ }^{\circ}$ AADvanced ${ }^{\mathrm{max}}$ positive) and apoptotic cells (the rest).

2.5. Statistical Analysis. Statistical analyses were conducted using the IBM SPSS 21.0 software. Kruskal-Wallis test followed by multiple comparisons was used to compare groups with different cell concentrations or in different storage solutions. Wilcoxon signed-rank test was used to compare fresh and thawed cells. Spearman correlation was used to evaluate the relationship between the two variables. $P<$ 0.05 was considered as statistically significant. Results are presented as mean \pm standard deviation (SD).

\section{Results}

3.1. Effect of Cell Concentration on Cell Clumping and Viability before Transplantation. No increase in the extent of cell clumping was observed with escalating concentrations of fresh rat BMMSCs in DPBS $(P>0.05)$. The viability (percentage of viable cells) was significantly higher $(P<0.001)$, while the corresponding numbers of dead and apoptotic cells were significantly lower $(P<0.001)$ in the $2 \times 10^{6} / \mathrm{mL}$ group than in the $0.2 \times 10^{6} / \mathrm{mL}$ group (Figure 3 ).

No significant correlation was found between the percentage of cell clumps and cell concentration $(r=-0.321$, $P>0.05)$. In contrast, the percentage of viable cells was positively correlated $(r=0.941, P<0.001)$, whereas the corresponding values of dead $(r=-0.831, P<0.001)$ and apoptotic cells $(r=-0.913, P<0.001)$ were negatively correlated with escalating cell concentrations.

3.2. Effects of Storage Solution and Storage Time on Cell Clumping and Viability. After harvesting from cell culture $(0 \mathrm{~h})$, there were significantly fewer cell clumps preserved in
NS ( $P<0.01$ compared to that of cells in DPBS or medium), with viability (>90\%), mortality, and apoptosis rates being comparable to those observed with cells in medium. The viability of fresh cells in DPBS was significantly reduced (>65\%), but the number of cell clumps was rather similar to that observed in medium (Figure 4).

Increasing the storage time increased cell clumping in medium $(r=0.635, P<0.001)$, but not in NS or DPBS $(P>$ 0.05) (Figure 4(a)). There was a time-dependent reduction in the viability in all three solutions, but this reduction was most pronounced in NS $(r=-0.963, P<0.001)$ and least evident in medium $(r=-0.726, P<0.001$ ) (Figure 4(b)). A time-dependent increase in the numbers of dead cells was also observed ( $r=0.668$ for the cells in medium, $r=0.969$ for the cells in normal saline, and $r=0.889$ for the cells in DPBS, $P<0.001$ ) (Figure 4(c)). There was a time-dependent increase in the numbers of apoptotic cells in medium $(r=$ $0.784, P<0.001)$ and a decrease in DPBS $(r=-0.745$, $P<0.001)$, but not in NS $(P>0.05)$ (Figure $4(\mathrm{~d}))$. The percentage of cells or clumps $>30 \mu \mathrm{m}$ in NS was positively correlated with the number of apoptotic cells $(r=0.410$, $P<0.05)$.

3.3. Effect of Freeze-Thawing Procedure on Cell Clumping and Viability. In comparison with the fresh cells, a significant increase of cell clumps after thawing was observed for the cells stored in NS $(P<0.05)$, whereas a decrease was observed for cells in DPBS $(P<0.05)$ (Figure 5(a)). There was a significant overall viability reduction of the thawed cells preserved in medium and NS ( $P<0.01$ for both groups), and a trend for those preserved in DPBS suspension (Figure 5(b)). The number of dead cells increased in all conditions $(P<$ 


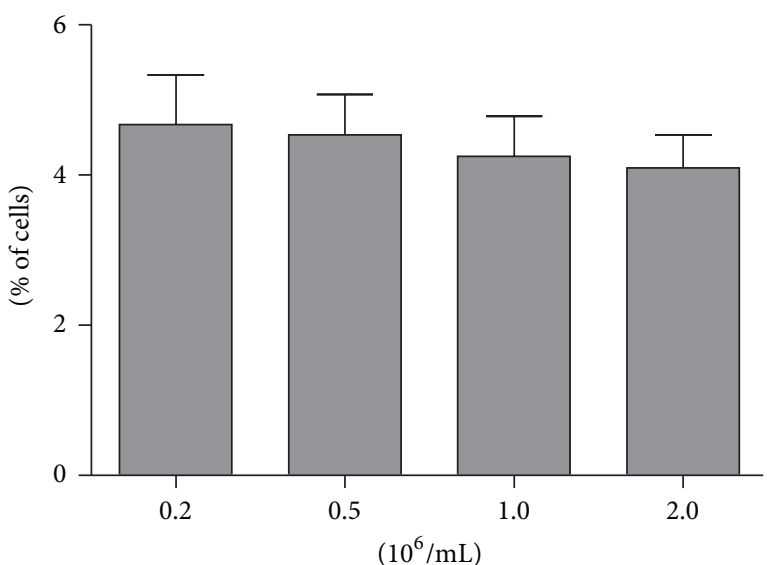

(a)

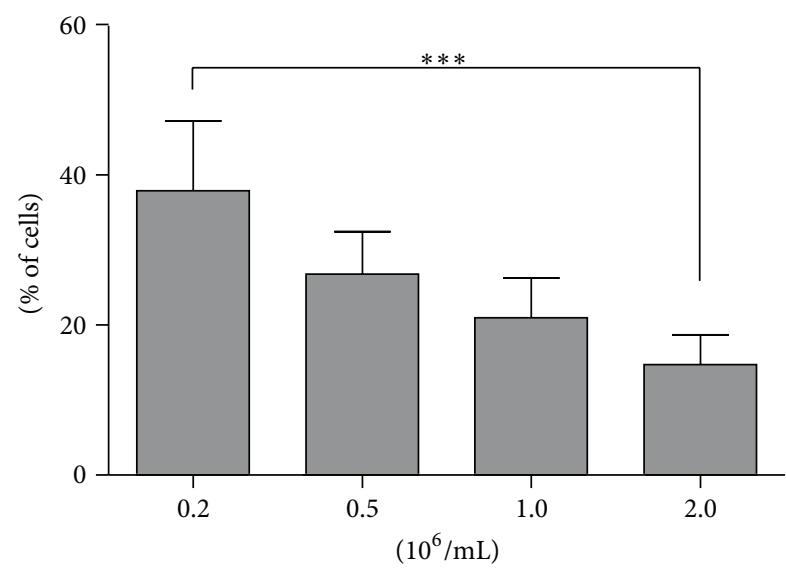

(c)

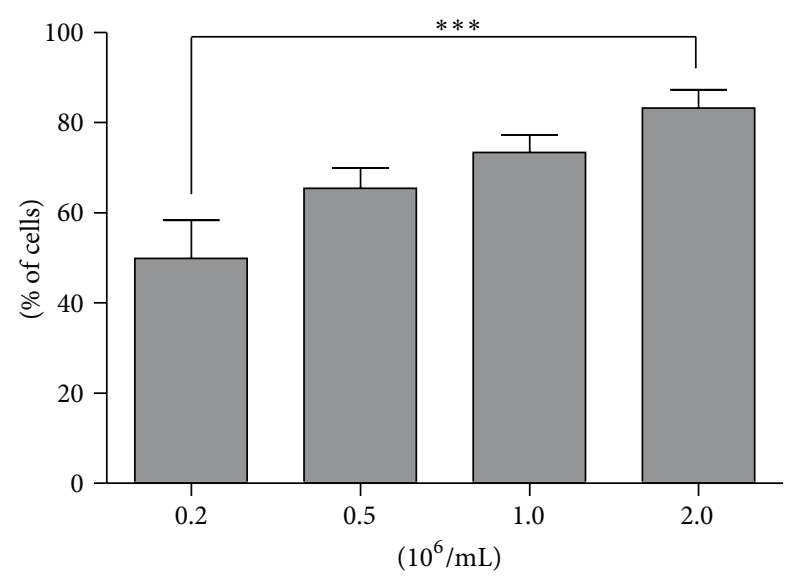

(b)

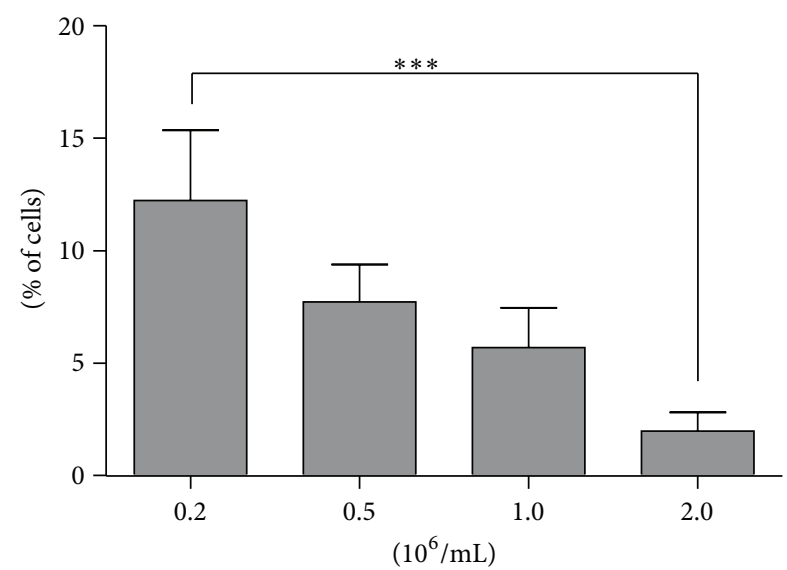

(d)

FIGURE 3: Effect of cell concentration on cell clumping and viability before transplantation. Fresh passage 5 rat BMMSCs were resuspended in $1 \mathrm{~mL}$ DPBS with escalating concentrations of $0.2,0.5,1.0$, and $2.0 \times 10^{6} / \mathrm{mL}$, respectively. (a) Cell clumps or large cells $>30 \mu \mathrm{m}$; (b) viable cells; (c) dead cells; (d) apoptotic cells. ${ }^{* * *} P<0.001$.

0.01 for cells in medium and NS, $P<0.05$ for cells in DPBS) (Figure 5(c)), and the number of apoptotic cells also increased for the cells in medium $(P<0.01)$ (Figure $5(\mathrm{~d})$ ).

The thawed cells stored in medium displayed the highest viability and the lowest percentage of dead cells $(P<0.05$ compared to the thawed cells in NS, $P<0.001$ compared to the thawed cells in DPBS) (Figures 5(b) and 5(c)); no significant difference was observed in the percentage of cell clumps or apoptotic cells (Figures 5(a) and 5(d)) across conditions.

\section{Discussion}

Currently there is an urgent need for the standardization of manufacturing, preparation, and transplantation of MSCs [17-19]. Our study provides new insights into MSC preparation by quantifying cell clumping in suspension prior to transplantation and for the first time investigated the effects of several common variables during the cell preparation procedure on both clumping and viability.

Our previous study observed a cell dose-related increase in cerebral embolism evoked after intra-arterial delivery into rats, using a constant volume of rat BMMSCs suspension in DPBS with different cell concentrations [12]. To exclude the possibility of increasing cell clumps with escalating cell concentration in suspension, cells in DPBS with concentrations from 0.2 to $2.0 \times 10^{6} / \mathrm{mL}$ were assessed in this study. In contrast to our expectations, the data clearly revealed that the degree of cell clumping in suspension did not increase with cell concentration. Therefore, this dosedependent cerebral embolism could have been associated with cellular interactions in vivo rather than the cell clumps in suspension per se. Increased cell aggregation in vivo, as well as the dose-dependent ability of MSCs to activate blood coagulation due to the expression of tissue factor and collagen type I after transplantation, could account for the cell doserelated safety issues [20,21]. Moreover, it is also possible that adhesion, cell clumping, and a change in the cell viability took place after delivery through the catheter and needle, but these issues were not evaluated in this study.

Cell viability increased with escalating cell concentrations, indicating that even a 10 -fold increase in cell concentration did not exert any direct negative impact on cell viability. However, it also needs to be emphasized that even the highest 

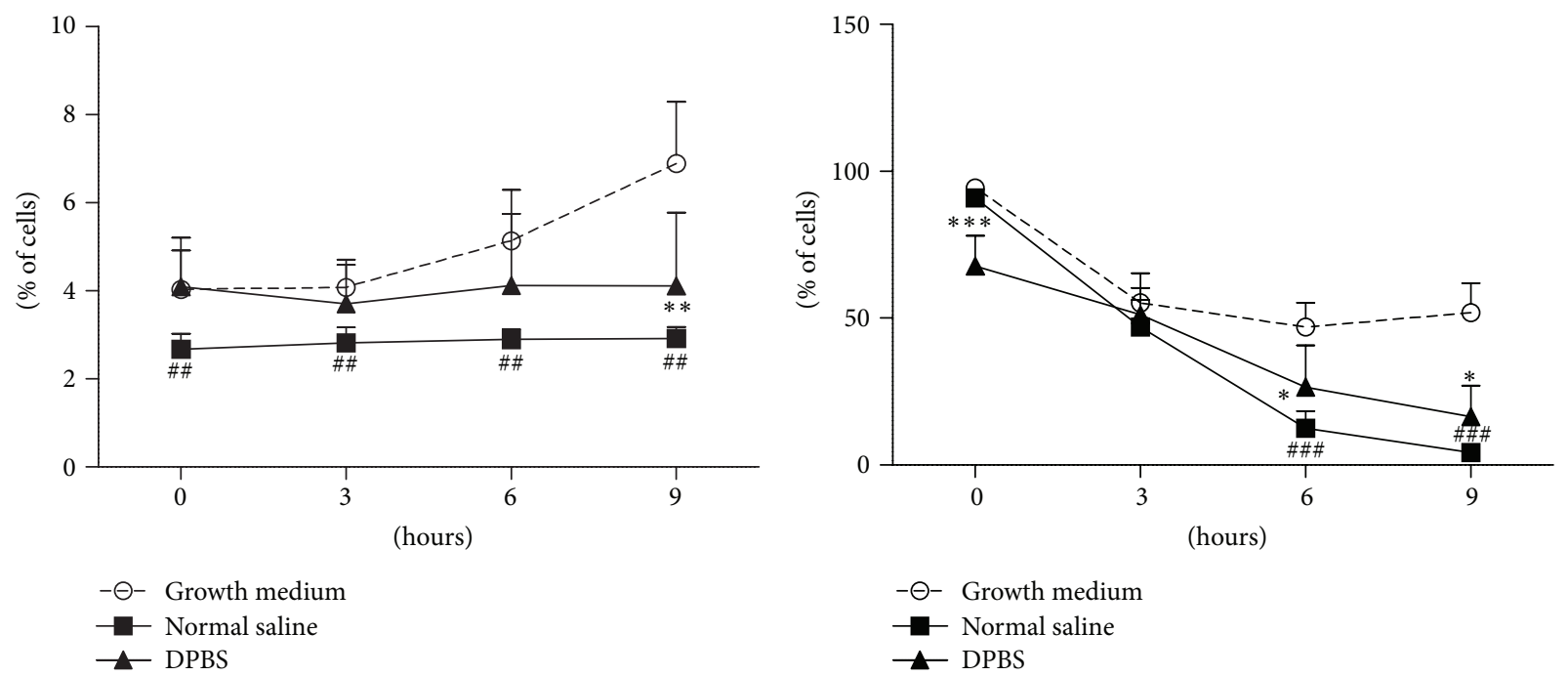

(a)
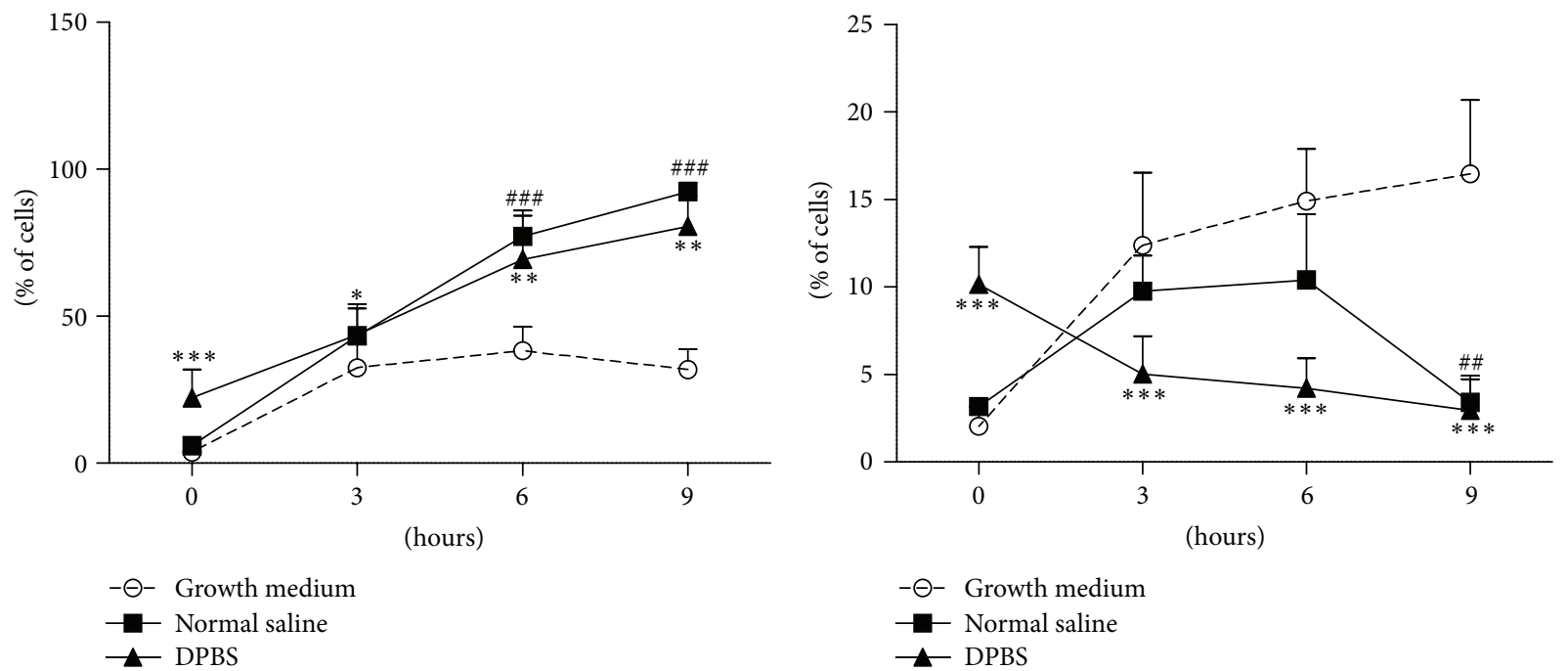

(c)

(d)

FIGURE 4: Effects of storage solution and storage time on cell clumping and viability before transplantation. Fresh passage 5 rat BMMSCs were resuspended in complete growth medium, normal saline, and DPBS and stored at $37^{\circ} \mathrm{C}$ for $3 \mathrm{~h}, 6 \mathrm{~h}$, and $9 \mathrm{~h}$ before analysis. (a) Cell clumps or large cells $>30 \mu \mathrm{m}$; (b) viable cells; (c) dead cells; (d) apoptotic cells. ${ }^{\# \#} P<0.01$ normal saline versus growth medium. ${ }^{\# \#} P<0.001$ normal saline versus growth medium. ${ }^{*} P<0.05$ DPBS versus growth medium; ${ }^{* *} P<0.01 \mathrm{DPBS}$ versus growth medium; ${ }^{* * *} P<0.001 \mathrm{DPBS}$ versus growth medium.

cell concentration $\left(2 \times 10^{6} / \mathrm{mL}\right)$ used in this study is still rather moderate, in comparison to the concentrations of up to $10 \times$ $10^{6} / \mathrm{mL}$ of MSCs reported via intra-arterial and $100 \times 10^{6} / \mathrm{mL}$ via intravenous injection [22, 23].

Phosphate-buffered saline, NS, and cell culture media have been the three most commonly used storage solutions before transplantation of MSCs in animal studies. Our data showed that, after harvesting, the percentage of cell clumps in NS was the lowest with $>90 \%$ viability. Before cell transplantation, up to several hours may elapse due to transportation and other technical reasons, and this time delay may affect the quality of the cell product. The numbers of cell clumps in medium increased with the storage duration in suspension, probably because the medium contains calcium and magnesium, which are important ions for cell adhesion. The viabilities of the cells in DPBS and NS decreased rapidly, probably due to the lack of nutrients or buffering capacity against exocytosed metabolic end products. Therefore, cells in solutions should be used immediately to ensure that there is neither any increase in cell clumping nor any reduction in cell viability. The number of cell clumps correlated with the number of apoptotic cells for the cells in NS, which might be related to the nucleic acids released by late apoptotic or dead cells.

The freeze-thawing procedure is another controversial factor that might affect cell quality. Cryopreserved cells are 


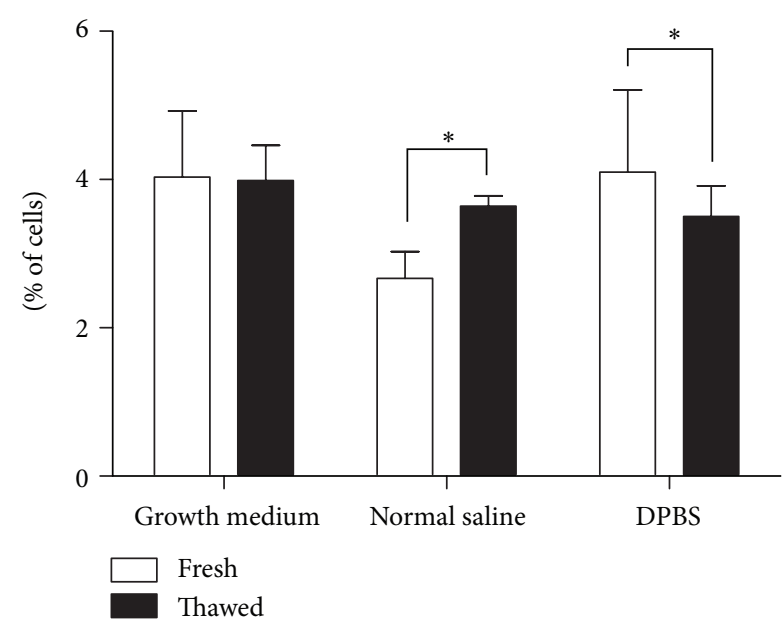

(a)

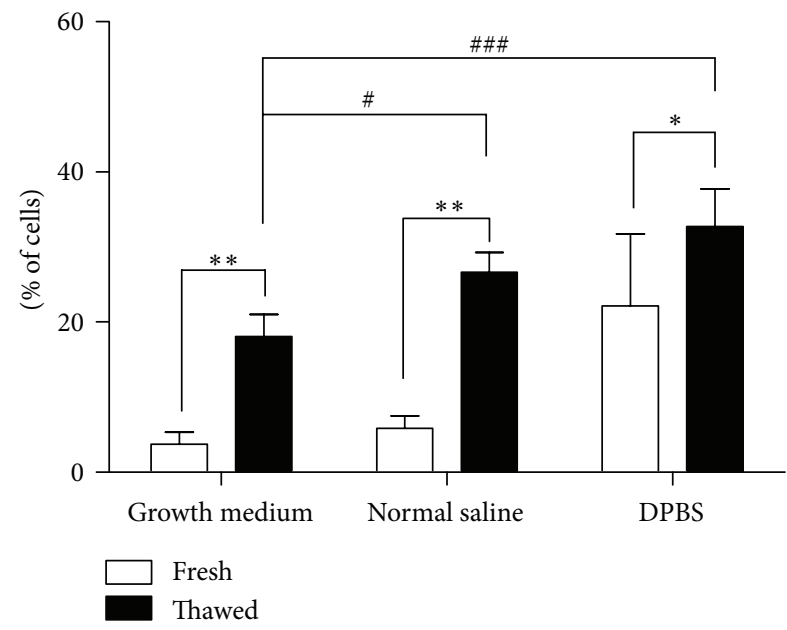

(c)

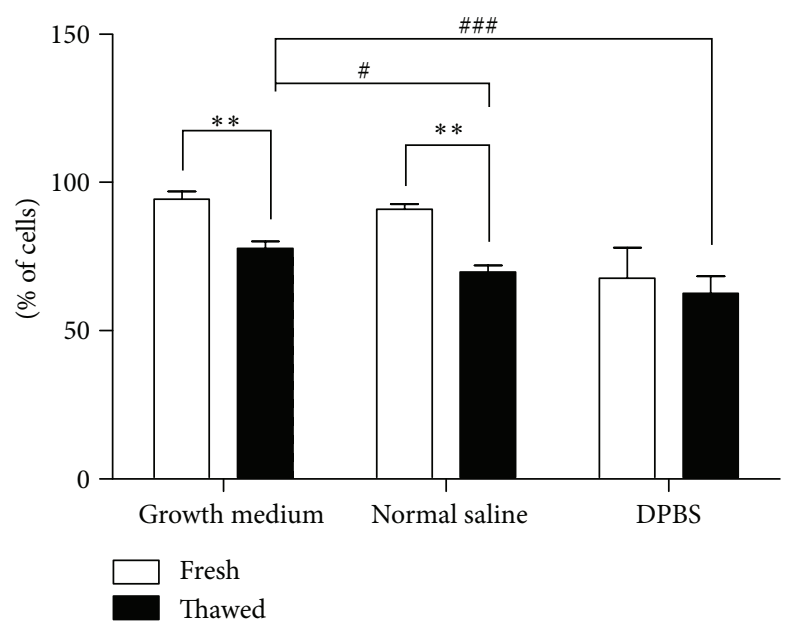

(b)

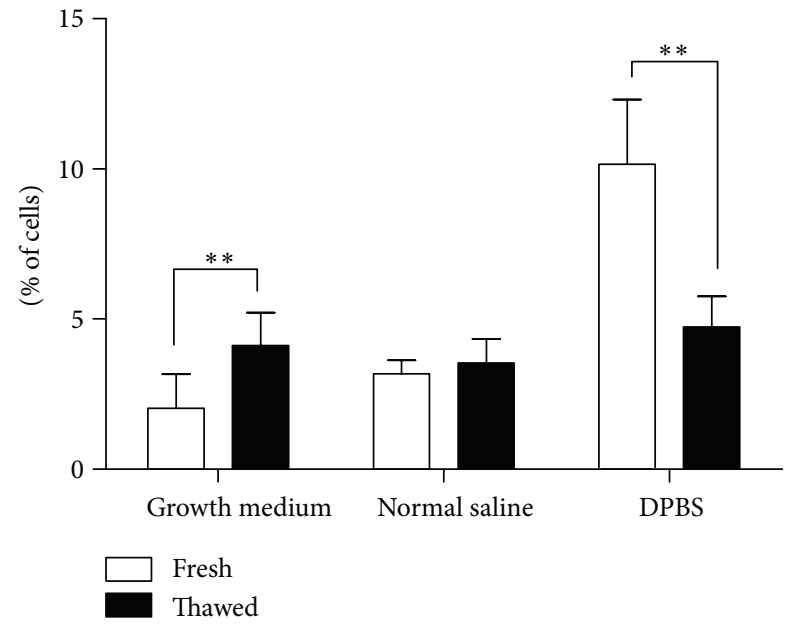

(d)

FIGURE 5: Effect of freeze-thawing procedure on cell clumping and viability of passage 5 rat BMMSCs. (a) Cell clumps or large cells $>30 \mu \mathrm{m}$; (b) viable cells; (c) dead cells; (d) apoptotic cells. ${ }^{*} P<0.05,{ }^{* *} P<0.01 ;{ }^{\#} P<0.05,{ }^{\# \#} P<0.001$.

good alternatives for cell therapy because of their immediate "off-the-shelf" availability. However, cryopreserved cells have been reported to exert compromised immunomodulatory effects and can induce a more intense immediate bloodmediated inflammatory reaction $[24,25]$, while some other recent studies have reported opposite findings [26, 27]. In this study, as expected, cryopreserved rat BMMSCs were less viable after thawing than their fresh counterparts with increasing numbers of dead and apoptotic cells, which might affect their therapeutic capabilities. Nevertheless, the changes of cell clumping for the cells in the three types of solutions were different after the freeze-thawing procedure, which could be related with different tendencies towards cell death and apoptosis and the corresponding buffering capacity.

An overestimation of cell viability with the trypan blue method (data not shown) as compared to flow cytometry results was observed in our study, consistent with previous findings from other groups $[24,25]$. This discrepancy in viability results is probably due to the presence of apoptotic cells that cannot be detected by trypan blue staining. Since viability results strongly rely on the method used, it is recommended that more sensitive and well-validated viability assays should be utilized in future preclinical and clinical studies of cell therapy.

The flow cytometry-based pulse-width assay provides a clear idea of size distribution and quantification of cell suspension and can also be used for the evaluation of filtering and other techniques to reduce clumps, recommending it as a potential release assay for clinical use. Criteria of clumps $\leq 5 \%$ of nondebris events and $\leq 150,000$ total particles $>30 \mu \mathrm{m}$ have been applied in one study using human bone marrow derived-aldehyde dehydrogenase bright cells, taking into account the number and diameters of human cerebral capillaries [14]. Similar criteria are not available for preclinical studies. However, due to the heterogeneity of preclinical results as well as the discrepancies observed between preclinical and clinical data, it would be advantageous if similar criteria could be devised for individual preclinical studies 
as this would ensure more consistent results and faster translation into the clinic.

\section{Conclusions}

Cell concentration, storage solution, storage time in suspension, and freeze-thawing procedure can influence cell viability and/or clumping before transplantation. The immediate use of fresh cells in NS seems to contain fewer cell clumps and also with high cell viability, both of which are beneficial for cell transplantation. Quantification of cell clumping can be conducted using the flow cytometry-based pulse-width assay prior to intravenous and particularly intra-arterial cell delivery in order to prevent vascular embolism.

$\begin{array}{ll}\text { Abbreviations } \\ \text { MSCs: } & \text { Mesenchymal stromal cells } \\ \text { BMMSCs: } & \text { Bone marrow derived mesenchymal } \\ & \text { stromal cells } \\ \text { FBS: } & \text { Fetal bovine serum } \\ \text { DPBS: } & \text { Dulbecco's phosphate-buffered saline } \\ \text { NS: } & \text { Normal saline } \\ \text { SD: } & \text { Standard deviation. }\end{array}$

\section{Conflict of Interests}

The authors declare that they have no competing interests.

\section{Acknowledgments}

The authors sincerely thank Marja Ahti for excellent technical assistance and Claudia Pösel for her critical comments on the paper. The study was supported by the Brain Research and Rehabilitation Foundation Neuron, ORION Research Foundation, and Finnish Cultural Foundation.

\section{References}

[1] X. Bao, J. Wei, M. Feng et al., "Transplantation of human bone marrow-derived mesenchymal stem cells promotes behavioral recovery and endogenous neurogenesis after cerebral ischemia in rats," Brain Research, vol. 1367, pp. 103-113, 2011.

[2] D. R. Yavagal, B. Lin, A. P. Raval et al., "Efficacy and dosedependent safety of intra-arterial delivery of mesenchymal stem cells in a rodent stroke model," PLoS ONE, vol. 9, no. 5, Article ID e93735, 2014.

[3] M. Janowski, D. C. Wagner, and J. Boltze, "Stem cell-based tissue replacement after stroke: factual necessity or notorious fiction?" Stroke, vol. 46, no. 8, pp. 2354-2363, 2015.

[4] J. Boltze, A. Arnold, P. Walczak, J. Jolkkonen, L. Cui, and D.-C. Wagner, "The dark side of the force-constraints and complications of cell therapies for stroke," Frontiers in Neurology, vol. 6, article 155, 2015.

[5] E. Eggenhofer, V. Benseler, A. Kroemer et al., "Mesenchymal stem cells are short-lived and do not migrate beyond the lungs after intravenous infusion," Frontiers in Immunology, vol. 3, article 297, 2012.
[6] K. Tatsumi, K. Ohashi, Y. Matsubara et al., "Tissue factor triggers procoagulation in transplanted mesenchymal stem cells leading to thromboembolism," Biochemical and Biophysical Research Communications, vol. 431, no. 2, pp. 203-209, 2013.

[7] V. Misra, M. M. Ritchie, L. L. Stone, W. C. Low, and V. Janardhan, "Stem cell therapy in ischemic stroke: role of IV and intraarterial therapy," Neurology, vol. 79, supplement 1, no. 13, pp. S207-S212, 2012.

[8] J. Lundberg, E. Södersten, E. Sundström et al., “Targeted intraarterial transplantation of stem cells to the injured CNS is more effective than intravenous administration: engraftment is dependent on cell type and adhesion molecule expression," Cell Transplantation, vol. 21, no. 1, pp. 333-343, 2012.

[9] P. H. Lee, J. W. Kim, O. Y. Bang, Y. H. Ahn, I. S. Joo, and K. Huh, "Autologous mesenchymal stem cell therapy delays the progression of neurological deficits in patients with multiple system atrophy," Clinical Pharmacology and Therapeutics, vol. 83, no. 5, pp. 723-730, 2008.

[10] P. Walczak, J. Zhang, A. A. Gilad et al., "Dual-modality monitoring of targeted intraarterial delivery of mesenchymal stem cells after transient ischemia," Stroke, vol. 39, no. 5, pp. 15691574, 2008.

[11] M. Janowski, A. Lyczek, C. Engels et al., "Cell size and velocity of injection are major determinants of the safety of intracarotid stem cell transplantation," Journal of Cerebral Blood Flow and Metabolism, vol. 33, no. 6, pp. 921-927, 2013.

[12] L. L. Cui, E. Kerkelä, A. Bakreen et al., "The cerebral embolism evoked by intra-arterial delivery of allogeneic bone marrow mesenchymal stem cells in rats is related to cell dose and infusion velocity," Stem Cell Research \& Therapy, vol. 6, article 11, 2015.

[13] J. Ge, L. Guo, S. Wang et al., "The size of mesenchymal stem cells is a significant cause of vascular obstructions and stroke," Stem Cell Reviews and Reports, vol. 10, no. 2, pp. 295-303, 2014.

[14] D. H. Hickerson, H. S. White, A. Nguyen et al., "Development of a flow cytometry-based pulse-width assay for detection of aggregates in cellular therapeutics to be infused by catheter," Cytotherapy, vol. 16, no. 11, pp. 1545-1557, 2014.

[15] C. V. Borlongan, S. Saporta, S. G. Poulos, A. Othberg, and P. R. Sanberg, "Viability and survival of hNT neurons determine degree of functional recovery in grafted ischemic rats," NeuroReport, vol. 9, no. 12, pp. 2837-2842, 1998.

[16] J. Khabbal, E. Kerkelä, B. Mitkari et al., "Differential clearance of rat and human bone marrow-derived mesenchymal stem cells from the brain after intra-arterial infusion in rats," Cell Transplantation, vol. 24, no. 5, pp. 819-828, 2015.

[17] J. M. Karp and G. S. Leng Teo, "Mesenchymal stem cell homing: the devil is in the details," Cell Stem Cell, vol. 4, no. 3, pp. 206216, 2009.

[18] The Stem Cell Therapies as an Emerging Paradigm in Stroke Participants, "Stem Cell Therapies as an Emerging Paradigm in Stroke (STEPS): bridging basic and clinical science for cellular and neurogenic factor therapy in treating stroke," Stroke, vol. 40, no. 2, pp. 510-515, 2009.

[19] S. I. Savitz, M. Chopp, R. Deans, S. T. Carmichael, D. Phinney, and L. Wechsler, "Stem cell therapy as an emerging paradigm for stroke (STEPS) II," Stroke, vol. 42, no. 3, pp. 825-829, 2011.

[20] Y. Fukuda, N. Horie, K. Satoh et al., "Intra-arterial transplantation of low-dose stem cells provides functional recovery without adverse effects after stroke," Cellular and Molecular Neurobiology, vol. 35, no. 3, pp. 399-406, 2015. 
[21] G. Moll, I. Rasmusson-Duprez, L. von Bahr et al., "Are therapeutic human mesenchymal stromal cells compatible with human blood?" Stem Cells, vol. 30, no. 7, pp. 1565-1574, 2012.

[22] L. Guo, J. Ge, Y. Zhou, S. Wang, R. C. H. Zhao, and Y. Wu, "Three-dimensional spheroid-cultured mesenchymal stem cells devoid of embolism attenuate brain stroke injury after intraarterial injection," Stem Cells and Development, vol. 23, no. 9, pp. 978-989, 2014.

[23] Y. Xu, S. W. Du, X. G. Yu, X. Han, J. C. Hou, and H. Guo, "Human bone marrow mesenchymal stem cell transplantation attenuates axonal injury in stroke rats," Neural Regeneration Research, vol. 9, no. 23, pp. 2053-2058, 2014.

[24] G. Moll, J. J. Alm, L. C. Davies et al., "Do cryopreserved mesenchymal stromal cells display impaired immunomodulatory and therapeutic properties?" Stem Cells, vol. 32, no. 9, pp. 24302442, 2014.

[25] M. François, I. B. Copland, S. Yuan, R. Romieu-Mourez, E. K. Waller, and J. Galipeau, "Cryopreserved mesenchymal stromal cells display impaired immunosuppressive properties as a result of heat-shock response and impaired interferon- $\gamma$ licensing," Cytotherapy, vol. 14, no. 2, pp. 147-152, 2012.

[26] F. F. Cruz, Z. D. Borg, M. Goodwin et al., "Freshly thawed and continuously cultured human bone marrow-derived mesenchymal stromal cells comparably ameliorate allergic airways inflammation in immunocompetent mice," Stem Cells Translational Medicine, vol. 4, no. 6, pp. 615-624, 2015.

[27] J. Luetzkendorf, K. Nerger, J. Hering et al., "Cryopreservation does not alter main characteristics of Good Manufacturing Process-grade human multipotent mesenchymal stromal cells including immunomodulating potential and lack of malignant transformation," Cytotherapy, vol. 17, no. 2, pp. 186-198, 2015. 

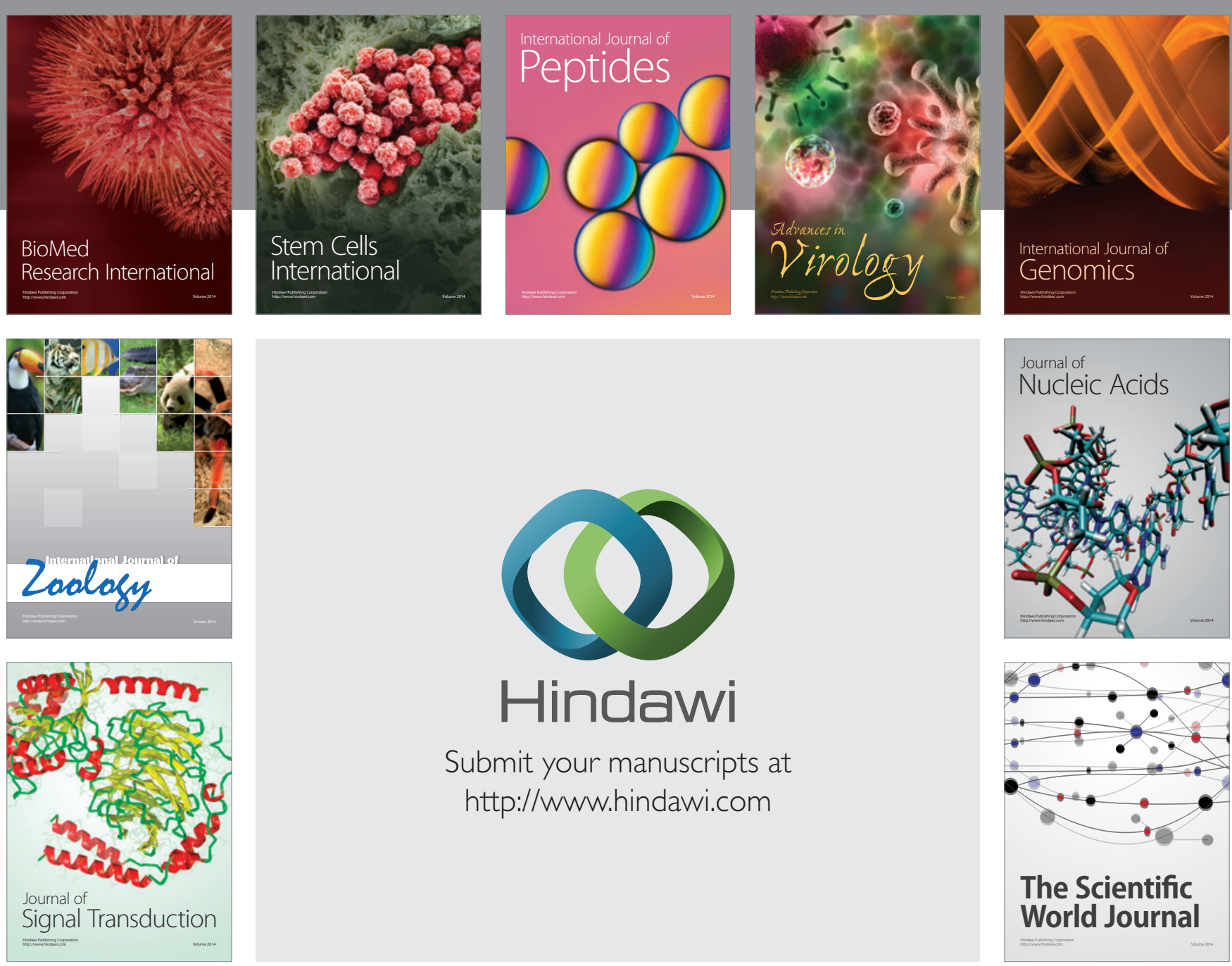

Submit your manuscripts at

http://www.hindawi.com
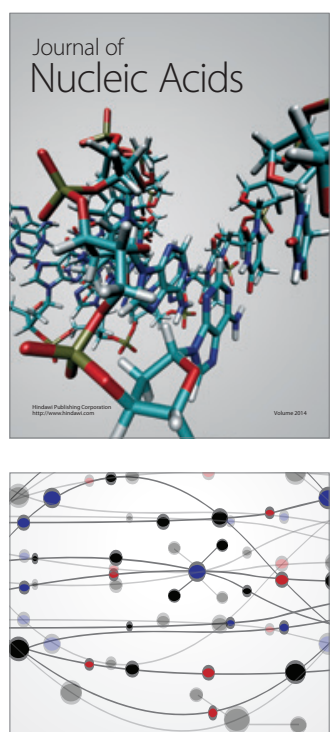

The Scientific World Journal
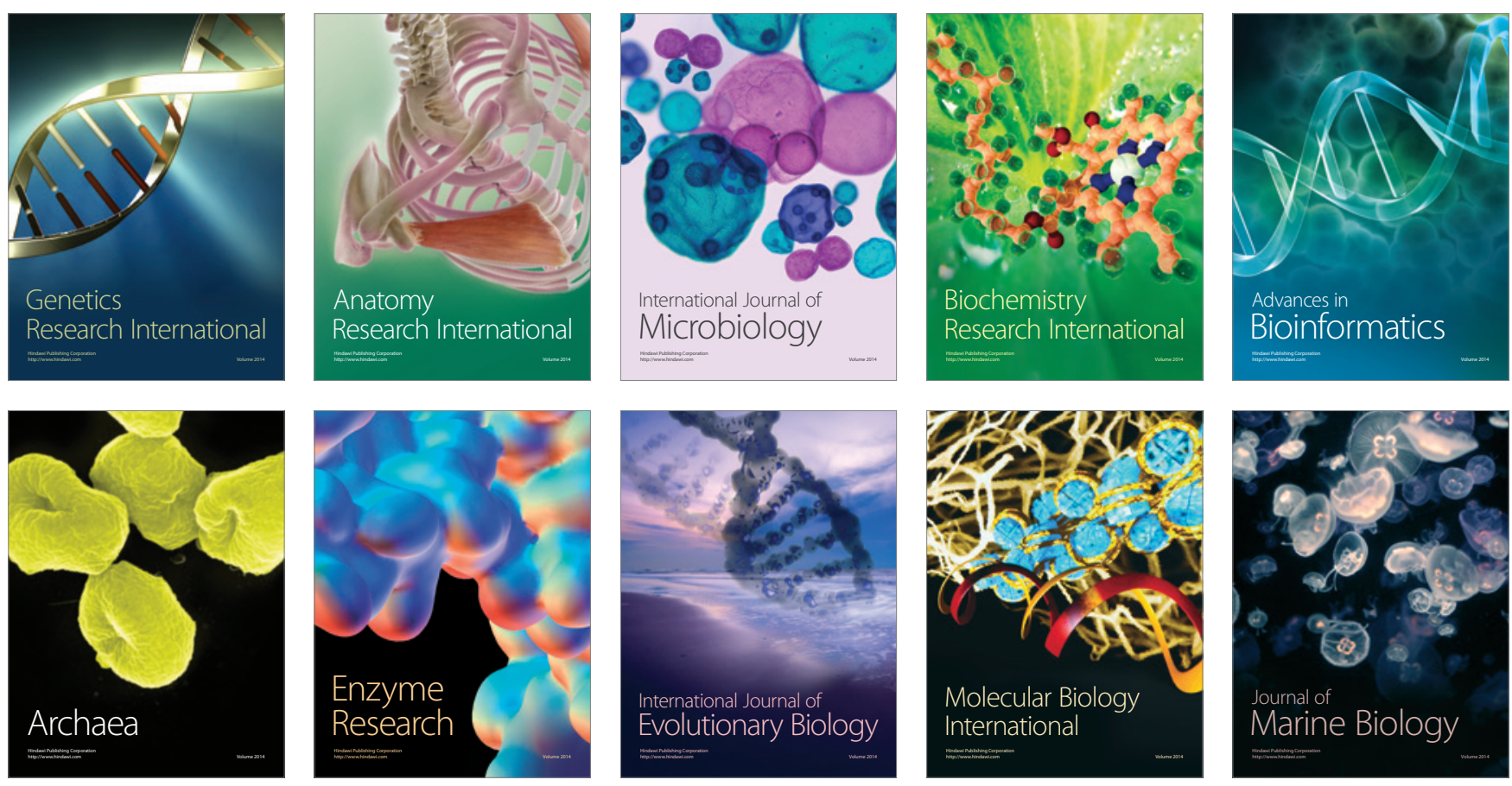